Bull Yamaguchi Med Sch 50(1-4) :29-37, 2003

\title{
Expression of CD80 and CD86 on Kupffer cells in the induc- tion of portal venous tolerance
}

\author{
Chizuru Sakamoto
}

First Department of Surgery, Yamaguchi University School of Medicine Yamaguchi, Japan

Key Words: CD80, CD86, portal venous tolerance, Kupffer cell

\begin{abstract}
For induction of an immune response, $\mathrm{T}$ cells should have been activated by signals mediated through costimulatory molecular such as CD80 (B7-1) and CD86 (B7-2) which being expressed on the surface of antigen presenting cells (APCs). The present study was designed to assess the rate of expression of CD80 and CD86 on recipient Kupffer cells (KCs), which have the abilities as APCs, under the state of both rejection and tolerance. For tolerant group, PVG rats received lymphocytes from DA rats were prepared. For iso and control group, PVG rats were inoculated by PVG spleen cells and phosphate buffered saline, respectively. After 1,3 or 7 days, inactivated spleen cells of DA rat were re-injected through caudal vein. The liver was extracted after perfusion. KCs were isolated by centrifugal elutriation. KCs were analyzed using flow cytometry after reacting with anti-rat FITC labeled CD80 or CD86 antibody. The expression rates of CD80 and CD86 in tolerant group were smaller than those in control and iso group $(\mathrm{p}<0.05)$. The results indicate the possibility that the immune hypo-responsiveness was contributed by downregulation of the costimulatory signals on $\mathrm{KCs}$, as a result of the intraportal inoculation of donor lymphocytes.
\end{abstract}

\section{Introduction}

Induction and maintenance of tolerance without aid of chemicals are the most preferable goal in clinical transplantation. Sultzberger $^{1)}$ and Chase $^{2)}$ showed that pretreatment of a target organ with an antigen could promote antigen-specific immune suppression of delayed-type hypersensitivity (DTH). Later, several investigators ${ }^{3,4)}$ have reported that donor lymphocyte inoculation into the portal vein of a recipient could induce donor-specific tolerance which, eventually, extended the organ allograft survival in animals. Induction mechanisms for such tolerance have been proposed by many authors but remains yet unsettled. Some authors ${ }^{5)}$ have suggested that KCs may participate in immune reaction in the liver so as to produce the donor-specific immune hypo-responsiveness.

$\mathrm{KCs}$ are resident hepatic macrophages which located in the hepatic sinusoids and have the ability of not only phagocytosis but presenting antigen (Ag) as antigen presenting cells (APCs) which activate T lymphocytes in the initiation of rejection of allogeneic grafts. ${ }^{6)}$ The stimulation of $\mathrm{T}$ lymphocytes is believed to require activation of two groups of intercellular signaling pathways. The first signal is derived from the ligation of the T-cell receptor (TCR) by Ag with class II major histocompatibility complex (MHC) molecules expressed on surface of APCs. 
The second signal is called costimulatory signal and mediated by the interaction of CD28 on $\mathrm{T}$ cells with $\mathrm{B} 7$ family ligands on the surface of APCs. CD28 shares its ligands (CD80 and CD86) with a related receptor (cytotoxic T lymphocyte antigen 4; CTLA4), which has been found to play a critical role in T-cell activation. ${ }^{7,8)}$ Inhibition of T-cell responses was achieved by using a soluble recombinant fusion protein of CTLA4 (CTLA4Ig) which blockades the CD28/B7 pathway. It was shown that costimulatory signals are important factor to produce T-cell-dependent immune responses. ${ }^{9)}$

Recently, it has been proved that KCs express B7 family on the surface similar to other APCs. ${ }^{10)}$ Therefore, we hypothesized that the lack of costimulatory molecules on KCs may play an important role in producing the portal venous tolerance. In the present study, we estimated the expression rates of CD80 and CD86 on KCs under the state of immune hyporesponsiveness resulting from the donor splenocytes administration via the portal vein.

\section{Materials and Methods}

Experimental Animals

Male PVG (RT1c) and DA (RT1a) rats (SLC Inc. Hamamatsu, Japan) weighing 225-250g were used as recipients and donors, respectively. This strain combination is fully allogeneic and results in acute transplant rejection. Animals were maintained under standard conditions in sterilized racks and received water and rodent chow ad libitum. PVG rats were divided into tolerant, iso and control group according to such the type of treatment that they received DA spleen cells, PVG spleen cells, and phosphate buffered saline (PBS), via the intraportal vein, respectively (Fig. 1). The study protocols were in compliance with institutional protocols.

Preparation of Allogeneic Spleen Cells (SPCs)

Rats were anesthetized with ether and intraperitoneal injection of pentobarbital sodium $(35 \mathrm{mg} / \mathrm{kg})$, same as in the following experiments.

After shaving and disinfection of the abdomen with $70 \%$ ethanol, an oblique subcostal incision was made. Spleen vessels were ligated before removal and minced in PBS.

\section{Experimental design}

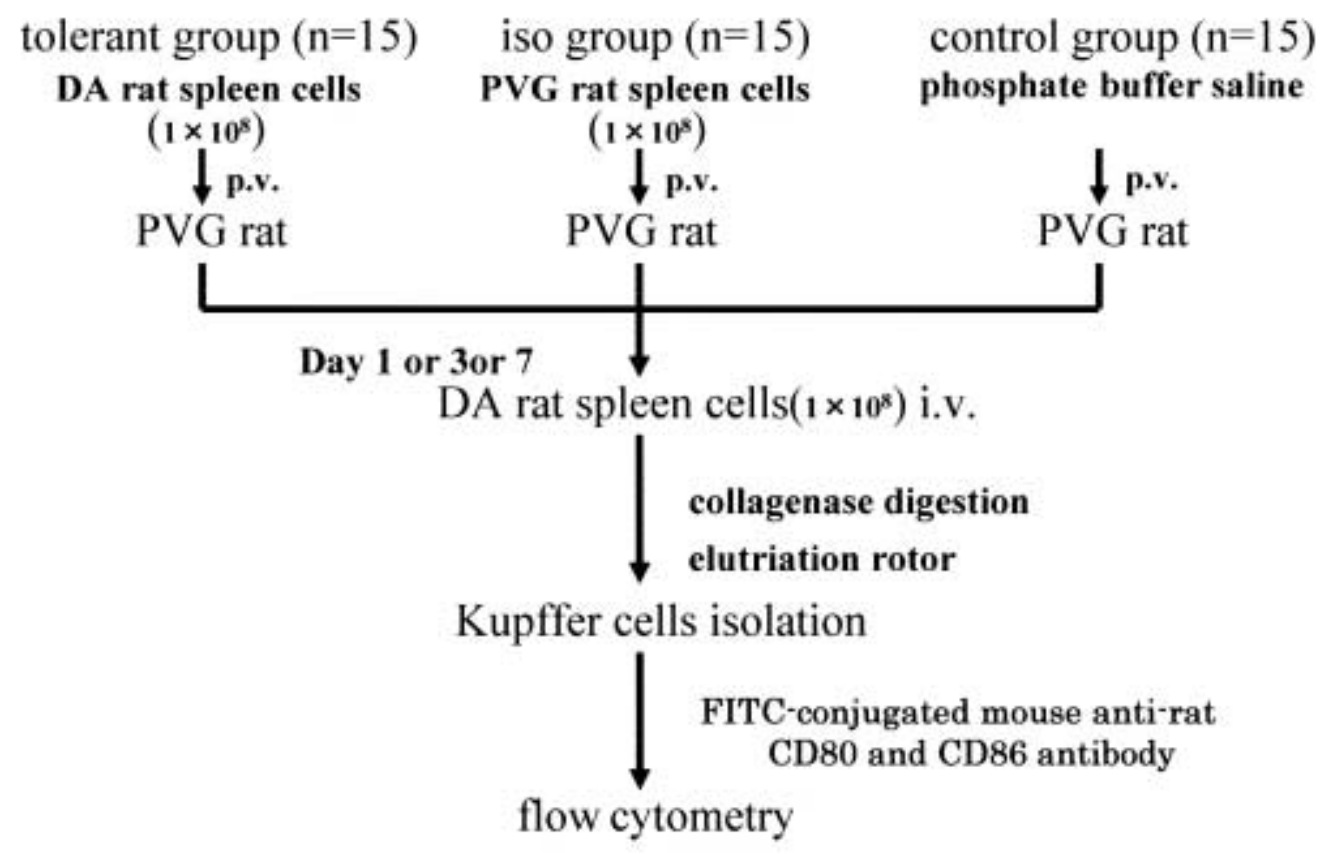

Fig. 1 Experimental design 
SPC suspension was prepared by passing through a nylon mesh after lysis of erythrocytes with ammonium chloride-Tris buffer and washed three times with PBS. Cell viability assessed by trypan blue exclusion test was always more than $90 \%$. The SPC concentration was adjusted to $1 \times 10^{8} / \mathrm{ml}$ in PBS for injection by intraportal route. ${ }^{3)}$

\section{Antigen Administration}

All animals were anesthetized with ether and pentobarbital sodium, as in the above. To expose the visceral organ, the abdominal skin was incised along the midline. SPCs were then injected through portal vein by a 26-gauge needle for tolerant and iso group, whereas control group received only PBS. At the end of injection, the needle was withdrawn while gently pressing the needling point with a cotton wool swab so as to achieve hemostasis without hematoma formation. The abdomen was then closed in two layers. ${ }^{4)}$

Second antigen administration was performed at day 1,3 or 7 using inactivated SPCs of DA rat. Before adjusting to $1 \times 10^{8}$ / $\mathrm{ml}$, these SPCs were treated by $100 \mu \mathrm{g} / \mathrm{ml}$ mitomycin C (Kyowahakko, Tokyo, Japan) for 30 minutes at $37^{\circ} \mathrm{C}$ and washed three times by $\mathrm{PBS}^{11)}, 1 \times 10^{8}$ inactivated SPCs were injected through caudal vein under anesthesia. After an hour, the following experiments were carried out.

\section{Cell Preparations}

KCs were isolated according to the method of previous studies. ${ }^{10,12,13)}$

The liver of anesthetized rat was perfused through portal vein with $\mathrm{Ca}^{2+}$ and $\mathrm{Mg}^{2+}$-free Hank's balanced salt solution (HBSS) containing $0.01 \%$ ethylene diaminetetraacetic acid (EDTA) and 1000 units of heparin, with a $\mathrm{pH}$ adjusted to 7.4. The liver was reperfused with HBSS containing $0.05 \%$ collagenase (Type-1; Nitta Zelatin, Osaka, Japan), 0.5mM calcium chloride, and $0.2 \%$ bovine serum albumin (BSA) (Sigma Chemical Co. St. Louis, $\mathrm{MO}, \mathrm{USA}$ ) at a flow rate of $12 \mathrm{ml} / \mathrm{min}$ at $37^{\circ} \mathrm{C}$. All the perfusates contained $20 \mathrm{mM}$ hydroxyethylpiperazine ethanesulfonic acid (HEPES). The liver was removed and teased apart in ice-cold HBSS containing 0.2\% BSA.
The cell suspension was poured through $70 \mu$ $\mathrm{m}$ nylon mesh and centrifuged at $45 \times \mathrm{g}$ for 2 min. The nonparenchymal cells rich in supernatant were collected. Centrifugal elutriator (BeckmanJ2-21 centrifuge and JE6-B rotor, Beckman Instruments, Palo Alto, CA, USA) was used for separation of nonparenchymal cells into fraction enriched for KCs. The cell suspension of KCs was centrifuged at $500 \times$ $\mathrm{g}$ for 10 minutes. The pellet was regulated $1 \times 10^{6}$ cells $/ \mathrm{ml}$ with PBS.

The purity and viability of KCs were more than $90 \%$ according to morphologic observation, immunostaining with antibody to the ED2 (Serotec Co., UK) and trypan blue exclusion test. The antibody ED2 recognizes a membrane antigen of tissue macrophages but have no reaction with monocytos, lymphocytes, granulocytos. ${ }^{14,15)}$

\section{Flow Cytometry}

Ten micrograms of monoclonal fluorsceinisothiocyanate (FITC) -conjugated mouse anti-rat CD80 (B7-1) antibody or CD86 (B72) antibody (Fujisawa Pharmaceutical Co., Osaka, Japan) was added to KCs. After 60 minnutes at $4^{\circ} \mathrm{C}$, the cells were washed twice with PBS and analyzed by flow cytometry (EPICS XL System II). Five thousand events per sample were collected and stored. The expression rates of CD80 or CD86 on $\mathrm{KCs}$ were analyzed.

\section{Statistical Analysis}

Results were expressed by mean \pm SE. To analyze statistical differences between each groups, Student's t test for equal variance was applied. p-values less than 0.05 were considered to be significant.

\section{Results}

The Expression Degree and Rate of CD80 (B7-1)

Fig. 2 demonstrates four representative histograms of KCs unstained (upper left) and stained with anti-CD80 in iso (upper right), torelant (lower left) and control (lower right) group. The intensity of fluorescence is iso and control group increased after staining with anti-CD80.

In Fig. 3, the expression rate of CD80 in control group (filled bars) was 14.28 \pm 


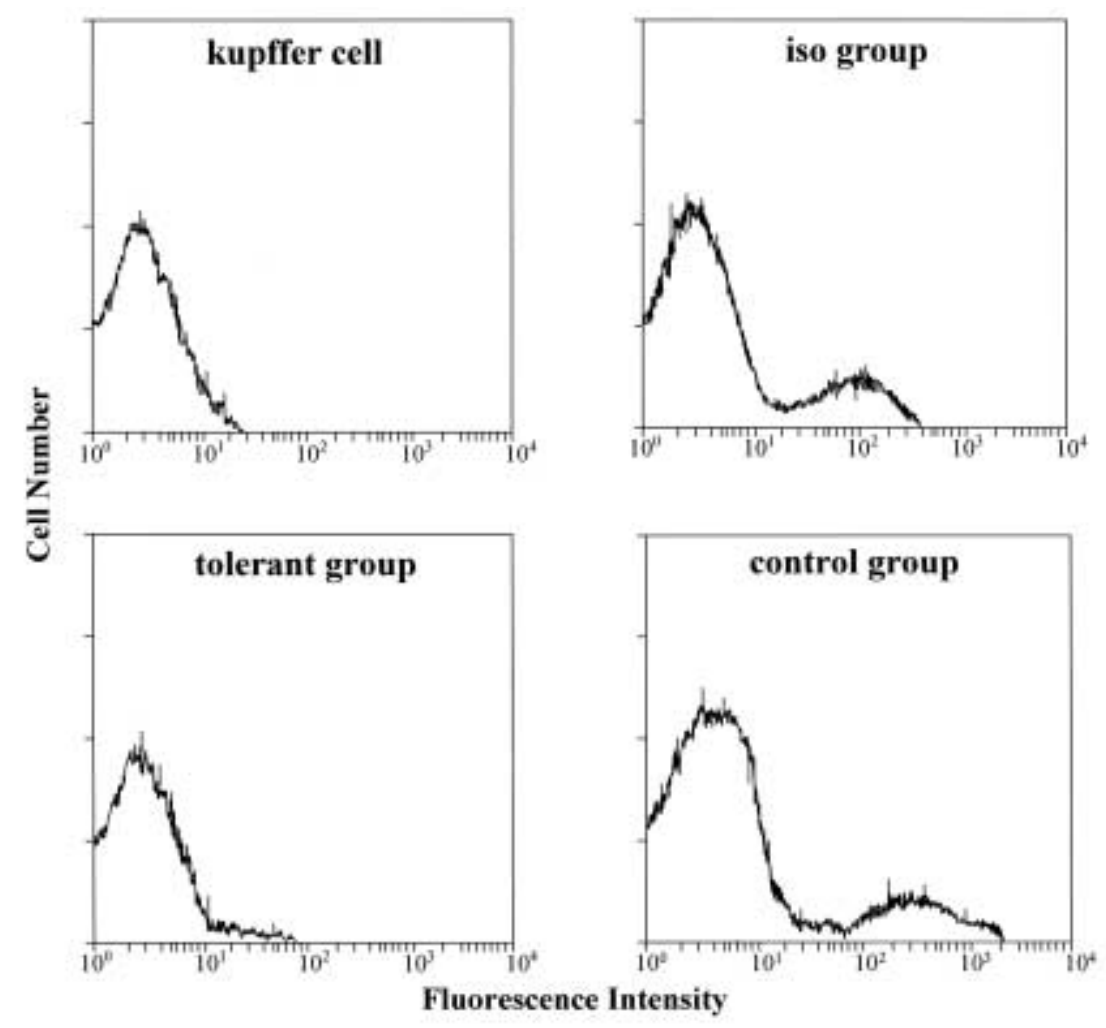

Fig. 2 Flow cytometric analysis of KCs, in iso, tolerant and control group, arranged from the upper left, to upper right, lower left and lower right, respectively. The histograms show CD 80 at day 7 .

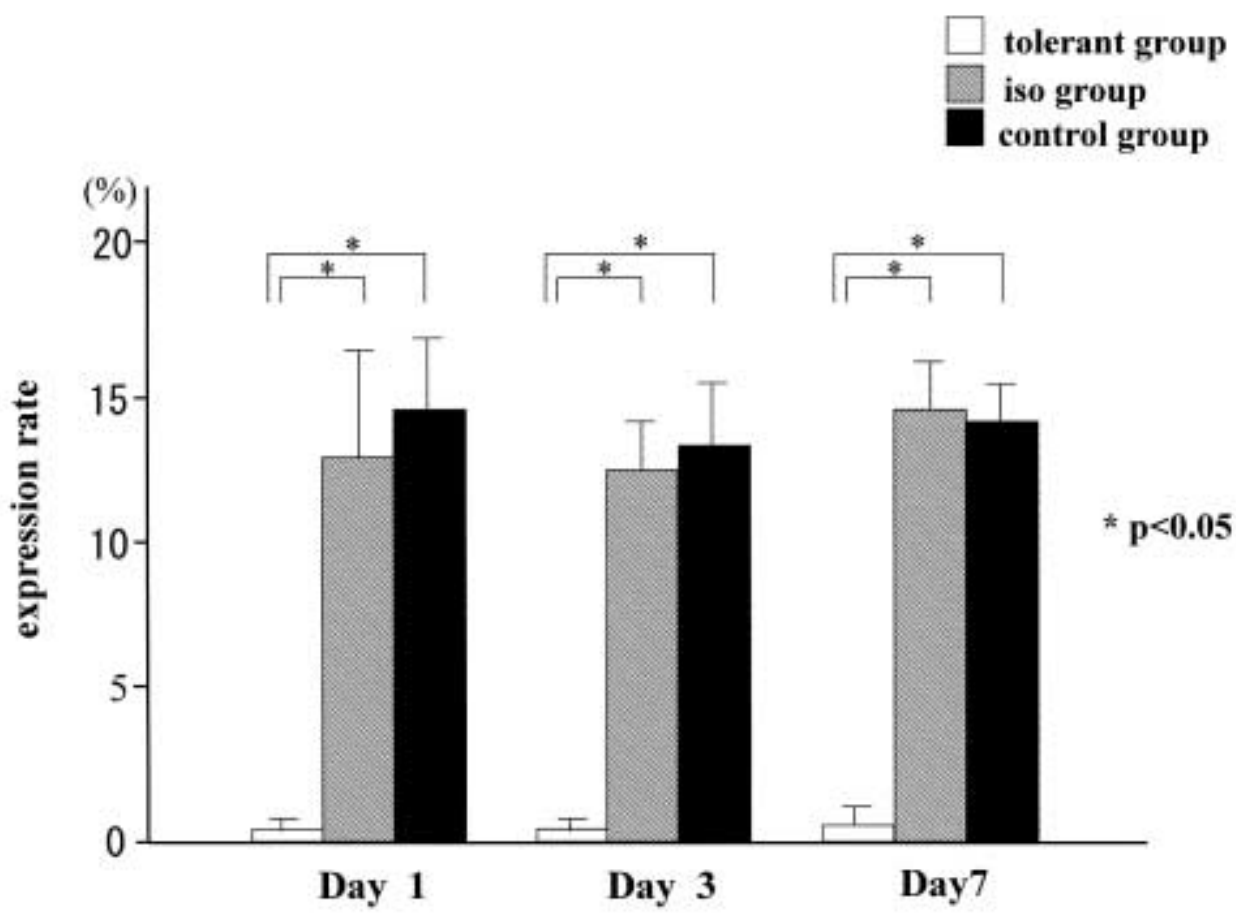

Fig. 3 Histograms exhibit the expression rates for $\operatorname{CD} 80$ at day 1,3 and 7, from left to right. The percentage of CD80 positive cells in tolernt group (empty bars) is significantly lower than those in iso (stripped ones) and control (filled ones) groups, when compared within the corresponding day. 
$2.92 \%$ at day $1,13.89 \pm 1.90 \%$ at day $3,14.02 \pm$ $3.49 \%$ at day 7 , whereas in iso group (stripped bars) that was $12.91 \pm 3.73 \%, 12.51 \pm 2.18 \%, 14$. $06 \pm 2.33 \%$, at the corresponding day. No significant difference in the expression rate of CD 80 between control and iso groups was found. On the contrary, the expression rate in tolerant group (empty bars) was $0.61 \pm$ $0.21 \%$ at day $1,0.39 \pm 0.16 \%$ at day $3,0.76 \pm 0$. $46 \%$ at day 7 . Thus there exists little variation in the expression rates with regard to the days after administration of antigen through portal vein, though in tolerance group the expression rates of CD80 are significantly lower $(p<0.05)$ than those in control and iso group at each day.

The Expression Degree and Rate of CD86 (B7-2)

In Fig. 4, four representative histograms of KCs are shown in the way similar to Fig. 2,

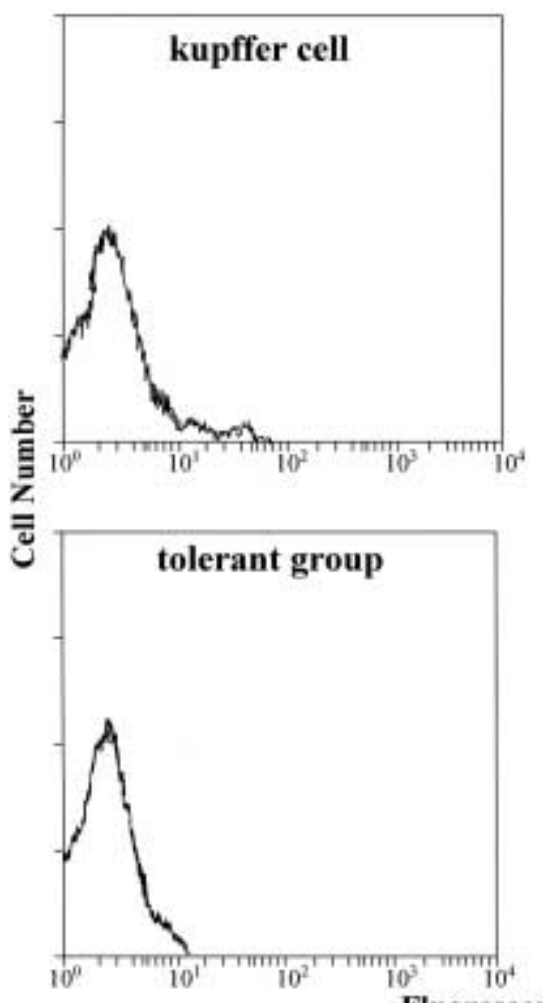

Fluorescence Intensity
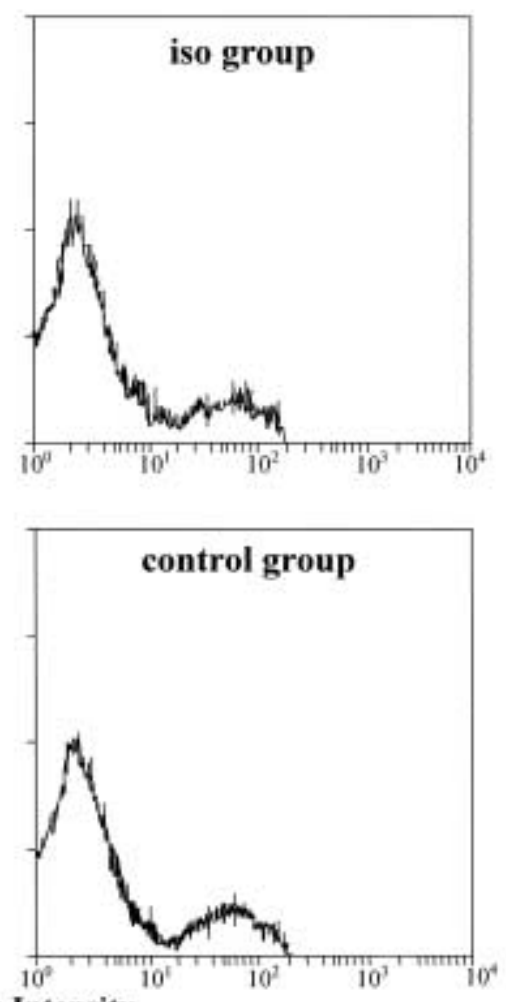

Fig. 4 Flow cytometric analysis similar to Fig. 2 but obtained from CD 86 at day 7.

\section{Discussion}

Donor specific-alloantigen transfusion (DST) could achieve antigen-specific tolerance and induce long-term graft survivals of but obtained with the use of anti-CD86 for staining. It illustrates that the intensity of fluorescence increased after staining $\mathrm{KCs}$ with anti-CD86 in iso and control group when compared with that of unstained. Note that in tolerant group the histogram is almost the same as that before staining.

As shown in Fig. 5, the expression rate for CD86 in tolerant group was $0.63 \pm 0.23 \%$ at day $1,0.35 \pm 0.18 \%$ at day $3,1.62 \pm 0.44 \%$ at day 7 , whereas in iso group it was $12.79 \pm 2$. $90 \%, 13.29 \pm 3.03 \%, 16.22 \pm 4.36 \%$ at the corresponding day and, in control group, it was 15 . $28 \pm 4.81 \%, 16.09 \pm 3.22 \%, 19.61 \pm 6.95 \%$, respectively. No significant difference was found between control and iso groups in the expression rate for $\mathrm{CD} 80$, though in tolerant group it was significantly reduced compared with the value in control and iso group $(\mathrm{p}<0.05)$. 
organ allograft survival than that resulting from inoculation through intravenous route.

Yoshimura $^{3)}$ reported intraportal administration of donor lymphocytes to recipient rats on the day of kidney transplantation, i.e. at day 0 , prolonged the survival of renal grafts, while intravenous administration caused extension of the mean survival rate slightly against untreated rats. The reason might depend on the counts of allogenic cells captured by the liver. In fact, Kenick et al. ${ }^{19)}$ demonstrated that all the cells administered via portal vein migrated into the liver, whereas $80 \%$ of the cells injected to the vein reached the liver.

Although the mechanism of systemic immunosuppression after portal venous inoculation of $\mathrm{Ag}$ is not yet clarified, it is known that after injection of radiolabeled allogenic cells into the portal vein, they were trapped by the hepatic component of reticuloendothelial system (RES). It implies that the process taken by RES in the liver is an initial requirement for the induction of tolerance. $5,19,20)$ In these previous studies, a most notable finding may be an existence of $\mathrm{KCs}$ and their role on the torelance. An early report suggests that KCs may be an essential factor of immunoreaction within the liver ${ }^{21}$. It was shown that portal venous tolerance could be produced after intravenous administration of gadolinium chloride, which is known to be a rare earth metal and is thought to prevent underlined tolerance by blocking KC's ability. ${ }^{22)} \mathrm{KC}$ has been known to have many such abilities that phagocytosis of foreign material, presentation of Ag, release of the immunosuppressive eicosanoid PGE2, which is known to inhibit IL-2 release by lymphocytes and prevents the ability of $\mathrm{Ag}$ presentation by $\mathrm{KCs}^{23,24)}$ and express also molecules such as class II MHC, B7 family.

Recently, it becomes evident that the immune response depends on an efficient $\mathrm{T}$ cell activation via the costimulatory molecules. The interaction of B7-1 (CD80) and B7-2 (CD 86) on APC with its counter-receptors CD28 on $\mathrm{T}$ cell surface generates a potent activating signal which amplifies the T cells. Namely the deficit of co-stimulation results in tolerance or anergy for specific antigen. CD80 is a 44 to $60-\mathrm{kd}$ member of the immunoglobulin superfamily with a limited expression on professional APC, and CD86 is a 75 to $115-\mathrm{kd}$ cell surface glycoprotein with $25 \%$ amino acid homology to CD80 expressed on APCs, too. ${ }^{25,26)}$

Our study showed CD80 and CD86 were found on the surface of KCs in iso and control groups, both being expected to induce rejection, while the tolerant group had little in appearance. Nakagawa etal. ${ }^{27)}$ alsodemonstrated that there existed a decrease in the expression of CD80/ CD86 and an increase in that of CTLA-4 antigen in the recipient liver which had been immunized by donor splenocytes administered via the portal vein.

This suggests that immune hypo-responsiveness by portovenous immunization can be achieved by KCs which express a decrease in CD80 and CD86. Futhermore because the expression rates of both CD80 and CD86 are decreased from the first day (day 1) after inoculation, the process should take place within 24 hours. The preceding study ${ }^{11)}$ also indicated that intraportal injection of donor native splenocytes on the day of transplantation could prolong the cardiac allografts in rats. Although the mechanism for this sequences is not yet clear, it is possible to explain in such a way, i.e., i), costimulatory blockade leads to differentiate native Th cell into Th2 subset that ii), releases some cytokines such as IL-10, an inhibitory action of which iii), on the expression of costimulatory molecules such as the B7 family has been well known. ${ }^{28)}$ Because the cytokine mentioned above is released not only by Th2 cells but also $\mathrm{KCs}^{29)}$, it is possible that when $\mathrm{KCs}$ were exposed initially by $\mathrm{Ag}$ mediated through the portal vein, CD80 and CD86 on $\mathrm{KCs}$ were consequently down-regulated by secretion of IL-10 from KCs and Th2.

It is shown that, in the results (Figs. 3, 5), a depression in the expression rates of CD80 and CD86 might occur within 1 day after the intraportal inoculation, even though induction of tolerance is considered to be accomplished on the day 7, after injection of antigen. In fact, Tanigawa et al. ${ }^{11)}$ described that cardiac allograft survivals were significantly prolonged when transplanted 10 days after intraportal injection of donor native SPCs, as compared with the result obtained immediately after transplantation on the day 
of administration. Prior to the present study, we prospected costimulatory blockade may promote gradually to cause the tolerance. However, our results (Figs. 3, 5) showed a marked decrease in the expression rates of CD80 and CD86, the degree of depression being not apparently associated with timing of the second antigen injection received within 7 days in tolerant group. The results (Figs. 2, $3,4,5)$ suggest that initial induction of tolerance may require some other factors in addition to depressing the expression rates of CD 80 and CD86 to a low level. Antigen-presenting function and B7 expression are existed not only in KCs but also in endothelial cells. ${ }^{10)}$ It is conceivable, therefore, that endothelial cells also participate in the immune reaction and tolerance together with KCs.

CTLA-4 was also considered to be one of the factors concerning the mechanism for the tolerance. The previous study demonstrated an increased expression of CTLA-4 antigen and a decreased expression of CD80 and CD86 on APCs in the recipient liver by splenocytes administered via the portal vein. ${ }^{27)}$
CTLA-4 is expressed on $\mathrm{T}$ cell and has stronger affinity for B7 family than CD28. If $\mathrm{T}$ cells don't recognize antigens on APCs for a low number of B7 molecules, CTLA-4 would be predominant in the immuno suppression by apoptosis induction or by reducing IL-2. ${ }^{30)}$

A question may arise that how long this effect can continue. Bolling et al. ${ }^{31)}$ reported that, for blocking CD28/B7 pathway completely and for maintaing the tolerance, DST alone is insufficient. However, they were able to obtain a remarkable prolongation of the cardiac allograft, when they utilized both CTLA4Ig and DST, the latter being mediated through the portal vein. Probably because, CTLA4Ig had interrupted the second signal by competitively inhibiting CD28 engagement and, hence, blocking activation of CD28. In short, DST itself may not be able to maintain the down-regulation of CD80 and CD86 for a long time. In further study, we have to confirm the duration of a decreased expression of both CD80 and CD86 in connection with the mechanisms for the portal venous tolerance.

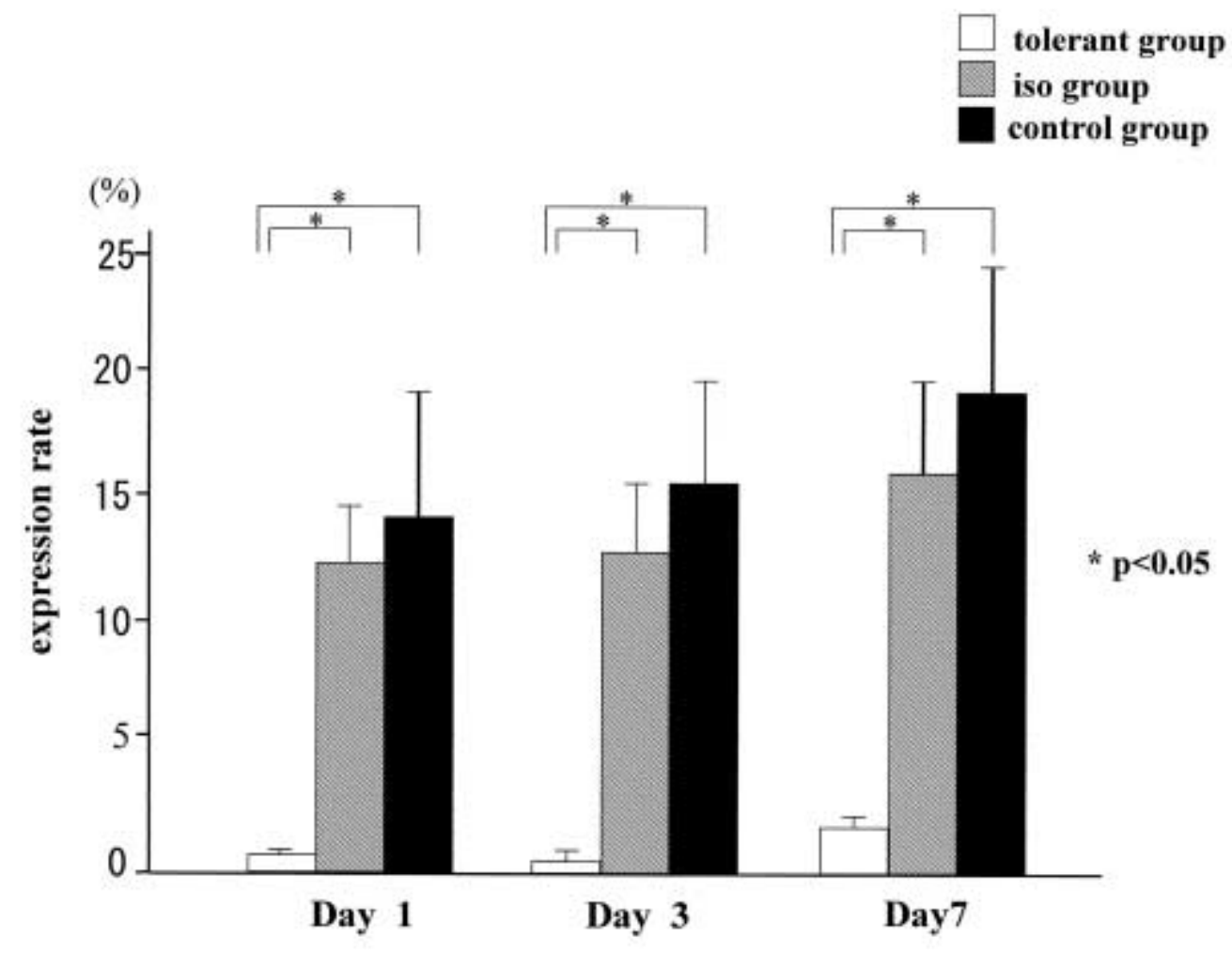

Fig. 5 Histograms similar to Fig. 3, but obtained from CD86. The expression rate in tolerant group is significantly lower than other groups at day 1 through day 7 . 


\section{Acknowledgement}

I gratefully thank Prof. K. Hamano for cordial discussions and advices on this study.

This work is partly supported by Grant in Aid for Scientific Research (1170696).

\section{References}

1) Sulzberger MB : Arsphename hypersensitiveness in guinea-pigs. Experiments demonstrating the role of skin,both as originator and as site of hypersensitiveness. Arch Dermatol Syphilol, 22 : 839, 1930.

2 ) Chase MW : Inhibition of experimental drug allergy by prior feeding of the sensitizing agents. Proc Soc Exp Biol med, $61: 257-259,1946$.

3 ) Yoshimura N, Matsui S, Hamashima T, Chol Joo Lee, Ohsaka Y and Oka T : The effects of perioperative portal venous inoculation with donor Lymphocytes on renal allograft survival in the rat. Trans ${ }^{-}$ plantation, 49 : 167-171, 1990.

4) Nakano Y, Monden M, Valdivia LA, Gotoh M, Tono $\mathrm{T}$ and Mori $\mathrm{T}$ : Permanent acceptance of liver allografts by intraportal injection of donor spleen cells in rat. Surgery, 111: 668-676, 1992.

5 ) Kamei T, Callery MP and Flye MW : Kupffer cell blockade prevents induction of portal venous tolerance in rat cardiac allograft Transplantation. J Surg Res, 48 : 393-396, 1990.

6 ) Wake K, Decker K, Kirn A, Knock DL, McCuskey RS, Bouwens L and Wisse E : Cell biology and kinetics of Kupffer cells in the liver. Int Res Cytol, 118 : 173229, 1989.

7 ) Allison JP:CD28-B7 interactions in T-cell activation. Curr Opin Immunol, $6: 414$ 419, 1994.

8 ) Thompson CB and Allison JP: The emerging role of CTLA4 as an immune attenuator. Immunity, $7:$ 445-450, 1997.

9 ) Linsley PS, Brady W, Urnes M, Grosmaire LS, Damle NK and Ledbetter JA : CTLA4 is a second receptor for the B cell activation antigen B7. J Exp Med, 174 : 561569, 1991.

10) Lohse AW, Knolle PA, Bilo K, Uhrig A,
Waldmann C, Ibe M,Schmitt E, Gerken $\mathrm{G}$ and Meyer zum Buschenfelde $\mathrm{KH}$ : Antigen-presenting function and B7 expression of murine sinusoidal Endotherial cells and Kupffer cells. Gastroenterology, 110 : 1175-1181, 1996.

11) Tanigawa $T$, Monden $M$, Nagano $H$, Hasuike Y, Gotoh M, Fukuzaki T, Ota M, Yoshida T and Mori T : Portal tolerance in rats: effect of donor cell treatment and timing of administration. Transplant Proc, 26 : 1870-1871, 1994.

12) Okamura K, Noshima S and Esato K : Cytokine release during hypoxia reoxygenation by Kupffer cells in rats with obstructive jaundice. Surg Today, $29: 730-734,1999$.

13) Maruiwa M, Mizoguchi A, Russell GJ, Narula N, Stronska M, Mizoguchi E, Rabb H, Arnaout MA and Bhan AK : Anti-KCA-3, a monoclonal antibody reactive with a rat complement $\mathrm{C} 3$ receptor, distinguishes Kupffer cells from other macrophage. J Immunol, 150 : 4019-4030, 1993.

14) Dijkstra CD, Dopp EA, Joling $P$ and Kraul G : The heterogeneity of mononuclear phagocytes in lymphoid organs : distinct macrophage subpopulations in the rat recognized by monoclonal antibodies ED1, ED2 and ED3. Immunology, 54 : 589-599, 1985.

15) Armburst $T$ and Ramadori $G$ : Functional characterization of two different Kupffer cell populations of normal rat liver. J Hepatol, 25 : 518-528, 1996.

16) Holman JM Jr and Todd R:Enhanced survival of heterotopic rat heart allografts with portal venous drainage. Transplantation, 49 : 229-230, 1990.

17) Sakai A : Role of the liver in kidney allograft rejection on the rat. Transplantation, $9: 333-334,1970$.

18) Schraut WH, Rosemurgy AS and Riddell RM : Prolongation of intestinal allograft survival without immunosuppressive drug therapy : transplantation of small bowel allografts. J Surg Res, 34 : 597-607, 1983.

19) Kenick S, Lowry RP, Forbes RD and Lisbona R : Prolonged cardiac survival following portal venous inoculation of allogenic cells: immunologically specific 
entrapment of allogenic cells within the liver. Transplant Proc, 19 : 3057, 1987.

20) Triger DR and Lloyd RS : Studies on hepatic uptake of antigen. II. The effect of hepatotoxin on the immune response. Immunology, 25 : 951, 1973.

21) Cantor HM and Dumont AE : Hepatic suppression of sensitization to antigen absorbed into portal system. Nature, $215: 744-745,1967$.

22) Roland CR, Mangino MJ, Duffy BF and Flye MW : Lymphocyte suppression by Kupffer cells prevents portal venous tolerance induction: a study of macrophage function after intravenous gadolinium. Transplantation, 55 : 11511158, 1993.

23) Rappaport RS and Dodge GR : Prostaglandin $\mathrm{E}$ inhibits the production of human interleuikin 2. J Exp $\mathrm{Med}$, 155: 943-948, 1982.

24) Ayala A, Perrin MM, Ertel $W$ and Chaudry IH : Differential effects of hemorrhage on Kupffer cells : decreased antigen presentation despite increased inflammatory cytokine (IL-1, IL-6 and TNF) release. Cytokine, $4: 66-75,1992$.

25) Chen C, Gault A, Shen L, Nabavi N: Molecular cloning and expression of early $\mathrm{T}$ cell costimulatory molecule- 1 and its characterization as CD86 molecule. $\mathrm{J} \mathrm{Im}^{-}$ munol, 152 : 4929-4936, 1994

26) Leifeld L, trautwein C, Dumoulin F, Manns M, Sauerbruch T, Spengler U : Enhanced expression of CD80(B7-1), CD 86(B7-2), and CD40 and their ligands CD
28 and CD154 in fulminant hepatic failure. Am J Path, 154 : 1711-1720, 1999

27) Nakagawa $K$, Matsuno $T$, Iwagaki $H$, Morimoto Y, Fujiwara T, Sadamori H, Inagaki $\mathrm{M}$, Urushihara $\mathrm{N}$, Yagi $\mathrm{T}$ and Tanaka N : Donor dendritic cells and recipient Kupffer cells in the induction of donor specific immune hyporesponsiveness. J Int Med Res, 29 : 119-130, 2001.

28) Buelens C, Verhasselt V, De Groote D, Thielemans K, Goldman M and Willems $\mathrm{F}$ : Human dendritic cell responses to lipopolysaccharide and CD40 ligation are differentially regulated by interleukin-10. Eur J Immunol, 27 : 1848-1852, 1997.

29) Knolle P, Schlaak J, Uhrig A, Kempf P, Meyer zum Buschenfelde $\mathrm{KH}$ and Gerken G : Human Kupffer cells secrete IL-10 in response to lipopolysaccharide (LPS) challenge. J Hepatol, 22 : 226-229, 1995.

30) Minguela A, Marin L, Torio A, Muro M, Garcia-Alonso AM, Moya-Quiles MR, Sanchez-Bueno F, Parrilla $\mathrm{P}$ and Alvarez-Lopez MR : CD28/CTLA-4 and CD80/CD86 costimulatory molecules are mainly involeved in acceptance or rejection of human liver transplant. Hum immunol, 61 : 658-669, 2000.

31) Bolling SF, Lin H, Wei RQ and Turka LA : Preventing allograft rejection with CTLA4Ig: effect of donor-specific transfusion route or timing. J Heart Lung Transplant, $15:$ 928-935, 1996. 